\title{
Summary of the updated international consensus diagnostic criteria for eosinophilic esophagitis: AGREE conference
}

\author{
Jonathan M. Spergel, MD, PhD ${ }^{1}$, Evan S. Dellon, MD, MPH ${ }^{2}$, Chris A. Liacouras, MD $^{3}$, Ikuo \\ Hirano, MD $^{4}$, Javier Molina-Infante, MD, PhD $^{5}$, Albert J. Bredenoord, MD, PhD ${ }^{5,6}$, and Glenn \\ T. Furuta, $M^{7}$ On behalf of the participants of AGREE
}

${ }^{1}$ Center for Pediatric Eosinophilic Diseases, Division of Allergy-Immunology, The Children's Hospital of Philadelphia, Perelman School of Medicine at Univ of Pennsylvania ${ }^{2}$ Center for Esophageal Diseases and Swallowing, Division of Gastroenterology and Hepatology, University of North Carolina School of Medicine, Chapel Hill, NC, USA ${ }^{3}$ Center for Pediatric Eosinophilic Diseases, Division of Gastroenterology and Hepatology \& Nutrition, Children's Hospital of Philadelphia, University of Pennsylvania Perelman School of Medicine ${ }^{4}$ Division of Gastroenterology and Hepatology, Northwestern University - Feinberg School of Medicine, Chicago, IL ${ }^{5}$ Department of Gastroenterology, Hospital Universitario San Pedro de Alcántara, Cáceres, Spain. Centro de Investigación Biomédica en Red Enfermedades Hepáticas y Digestivas (CIBEREHD), Madrid, Spain ${ }^{6}$ Department of Gastroenterology, Academic Medical Center, Amsterdam, The Netherlands ${ }^{7}$ Digestive Health Institute, Children's Hospital Colorado, Aurora, CO; Gastrointestinal Eosinophilic Diseases Program, University of Colorado School of Medicine, Aurora, CO

\section{INTRODUCTION}

EoE is a global health condition that has steady increased over the last few decades and is now reported on all continents, with an estimated incidence of 4 per 100,000. Diagnostic Guidelines on eosinophilic esophagitis (EoE) were published in 2007 and updated in 2011. ${ }^{1,2}$ EoE is defined as a clinicopathological condition that is likely immune or antigen driven, and characterized clinically by symptoms of esophageal dysfunction and histologically by $\geq 15$ eosinophils per high power field (eos/hpf). In the initial guidelines, inflammation related to gastro-esophageal reflux disease (GERD) needed to be ruled out as an underlying cause and expert consensus determined that the best approach would be to use either high dose proton pump inhibitor (PPI) treatment or $\mathrm{pH}$ monitoring. EoE and GERD were felt to be mutually exclusive disorders where response to a PPI trial/pathologic $\mathrm{pH}$ exposure was consistent with GERD and non-response/normal $\mathrm{pH}$ monitoring confirmed

Corresponding Author: Jonathan M. Spergel, MD, PhD, The Children's Hospital of Philadelphia, Division of Allergy and Immunology, 3401 Civic Center Blvd. Wood Building, $3^{\text {rd }}$ floor, Philadelphia, PA 19104, Phone: (215) 590-2549, Fax: (215) 590-6849, spergel@email.chop.edu.

Publisher's Disclaimer: This is a PDF file of an unedited manuscript that has been accepted for publication. As a service to our customers we are providing this early version of the manuscript. The manuscript will undergo copyediting, typesetting, and review of the resulting proof before it is published in its final citable form. Please note that during the production process errors may be discovered which could affect the content, and all legal disclaimers that apply to the journal pertain. 
EoE. However, multiple investigators observed that a large proportion of patients with clinical symptoms suggestive of EoE and esophageal eosinophilia $\geq 15$ eos/hpf responded to treatment with high-dose PPI. ${ }^{3}$ Even in these reports, there is heterogeneity and inconsistency of the definition of high-dose PPI. Because of this, diagnostic guidelines published in 2011, 2013, and 2014 defined a new condition termed PPI-responsive esophageal eosinophilia (PPI-REE) ${ }^{2}$ Lack of response to a PPI trial confirmed the diagnosis of EoE, and response was suggestive of either GERD or PPI-REE.

An evolving body of research suggested that EoE and GERD were not mutually exclusive and instead had a complex relationship. In addition, multiple retrospective and prospective studies found that esophageal eosinophilia in patients with symptoms and endoscopic signs suggestive of EoE can respond to PPI therapy in both children and adult at a rate of $28-82 \%$ (summarized in Dellon et al, Gastroenterology paper) ${ }^{3}$. In a meta-analysis by Lucendo and colleagues assessing PPIs for treatment of symptomatic esophageal eosinophilia, the pooled histologic response to PPI was about 50\% although heterogeneity was high $\left(\mathrm{I}^{2}=66 \%\right){ }^{4}$ Therefore, it is estimated about $50 \%$ of the patients with esophageal eosinophilia respond to a PPI and those who do not respond to a PPI, almost all (>90\%) in children and adult respond to an elemental diet. However, how many patients who respond to a PPI also respond to diet is an unanswered and important question (Figure 1).

In addition, similar studies examined the clinical, endoscopic, and histologic features at baseline (prior to a PPI trial) of both EoE and PPI-REE cases, and found that few, if any, of these factors that could distinguish the two. ${ }^{5,6}$ However, recent study found an increase in IgE-mediated food allergy in EoE population compared to PPI-REE ${ }^{7}$. In addition, RNA expression profiles were largely similar between the two conditions (and distinct from GERD), though some differences were seen particularly related to a potassium channel in PPI-REE ${ }^{8,9}$ Finally, there are two case reports that some individuals respond to both diet and PPI. ${ }^{10,11}$

Potential non-acid mediated anti-inflammatory mechanisms of PPIs were identified. ${ }^{12-14}$ For example, PPIs inhibit the expression of intercellular adhesion molecule-1 (ICAM-1) and vascular cell adhesion molecule-1 (VCAM-1), an adhesion molecule recognized by ligands on the eosinophil cell surface. ${ }^{15}$ PPIs also block IL-13 and IL-4 stimulated increase in eotaxin-3 mRNA expression and protein secretion, ${ }^{12}$ via a STAT6 (signal transducer and activator of transcription 6) binding to the eotaxin-3 promoter. ${ }^{13}$ Finally, PPIs may improve the epithelial barrier function in the esophagus. ${ }^{16,17}$

Overall, these findings raised an important question of whether PPI-REE is a subset of EoE in both pediatric and adults. A new European EoE guideline, published in 2017, ${ }^{18}$ suggested that PPI-REE and EoE were on the same spectrum and that PPIs could be considered a treatment for EoE. To develop an international consensus, the AGREE (A working Group on ppi-REE) Conference was held on May 6, 2017 in Chicago IL. The full report is published in Gastroenterology and the following are highlights of the report. 


\section{New Diagnostic Criteria}

EoE is suspected on a clinical basis with chronic symptoms of esophageal dysfunction (dysphagia, food impaction, food refusal, failure to progress with food introduction, heartburn, regurgitation, vomiting, chest pain, odynophagia, abdominal pain, failure to thrive, among others). The updated diagnostic algorithm for EoE is shown in Figure 2, based on the diagnostic criteria listed in Table 1.

When endoscopy is performed, the esophagus should be examined for features of EoE (including esophageal rings, longitudinal furrows, exudates, edema, strictures, or narrow caliber esophagus, ideally quantified using the EoE Endoscopic Reference Score $[\text { EREFS] })^{19}$ as well as alternative esophageal disorders. In all cases, including when the esophagus looks normal, esophageal biopsy specimens should be obtained with multiple biopsies from two or more esophageal levels. ${ }^{1,2,20}$ Gastric and duodenal biopsies should be obtained as clinically indicated by symptoms.

At this stage in the algorithm, a patient would be considered to have clinically suspected $\underline{\text { EoE }}$ if there are symptoms of esophageal dysfunction and at least 15 eos/hpf (or 60 eos $/ \mathrm{mm}^{2}$ ) on biopsy. There may be patients who enter the algorithm at this step, those who have abnormal biopsies, even if EoE was not a clinical consideration prior to the endoscopy and biopsy. A key point is that the presence of esophageal eosinophilia on histologic examination without further consideration of the clinical presentation is not diagnostic of EoE.

Because of the data discussed above, a PPI trial is not required for diagnosis of EoE in this algorithm. However, use of concomitant therapies must be considered when interpreting endoscopy and biopsy results. Often, patients who have symptoms that may be related to GERD or other acid related disorders are first treated with a PPI, and if they cannot stop this medication without a recurrence of symptoms, an EGD is performed while they are taking a PPI. Thus, a diagnosis of EoE cannot be definitively ruled out in patients who have an initial endoscopy for evaluation of symptoms suggestive of EoE but who are on a PPI and who have normal biopsies, because their biopsy results in the absence of PPI therapy are not known. For patients who respond to PPI therapy, clinicians must eventually decide whether ongoing long-term PPI therapy should be utilized or whether further evaluation off of PPI therapy should be considered. Conversely, patients on PPI treatment when they come to endoscopy may have endoscopic findings and biopsies consistent with EoE, but still need to follow-through with the remainder of the diagnostic algorithm.

All patients with esophageal eosinophilia of $\geq 15$ eos/hpf $\left(\sim 60 \mathrm{eos} / \mathrm{mm}^{2}\right)$ should be evaluated for non-EoE disorders that cause or potentially contribute to esophageal eosinophilia. Hypereosinophilic syndrome, non-EoE EGIDs, Crohn's disease, infections, connective tissue disorders and drug hypersensitivity reactions (see Table 1) have been associated with esophageal eosinophilia but are uncommon or present with clinical features that readily distinguish them from EoE. ${ }^{2}$ GERD continues to present a unique situation and is discussed in detail in the Gastroenterology paper. In some patients, it may be difficult to ascertain the precise contribution of GERD to esophageal eosinophilia, and clinical evaluation for GERD 
could be undertaken in concordance with published GERD guidelines prior to a definitive diagnosis of EoE. ${ }^{21}$

EoE is finally diagnosed after evaluation shows there are no other etiologies substantially contributing to symptoms and esophageal eosinophilia. We define confirmed EoE as symptoms of esophageal dysfunction, at least $15 \mathrm{eos} / \mathrm{hpf}$ ( or $\sim 60 \mathrm{eos} / \mathrm{mm}^{2}$ ) on biopsy, and evaluation showing no significant other causes of symptoms and/or esophageal eosinophilia. We recommend that providers understand that the diagnostic criteria are and always have been uniform with respect to patient age, and that all providers involved in the diagnosis (allergist, gastroenterologist, pathologist and researchers) approach the patient with potential EoE without any age-based bias.

Initial treatment and follow-up after EoE is confirmed-It is beyond the scope of this paper to provide comprehensive recommendations for the treatment of EoE. ${ }^{18,22}$ To date, no prospective double-blind randomized trial has compared the efficacies of steroids to PPI, or diet to PPI. However, due to low cost, good safety profile, convenience, and a large body of literature describing response rates in patients with esophageal eosinophilia, a PPI should be considered as a potential initial treatment (especially if it has not been used before), although steroids or dietary elimination may also be considered. ${ }^{4,}{ }^{18}$ Due to the fact that single arm observational studies have found modest response rate to PPI in patients with esophageal eosinophilia. If diet or steroid therapy is used as a first line therapy and is determined to be ineffective on follow-up studies including endoscopy with biopsy, PPI therapy should be considered as there is a good chance that this will be successful. It is also necessarily to realize that because GERD and EoE may coexist, some patients may need to be treated with both a PPI and different anti-inflammatory treatment (e.g. dietary elimination or a topical steroids) in order to optimally treat both conditions, though there are few data on combination therapy. Finally, treatment decisions must be made with the understanding that the majority of data on response rates for topical steroids, dietary elimination, and novel/ emerging treatments in EoE are almost entirely in the patient population that has failed to respond to PPI treatment previously. ${ }^{22}$

\section{Conclusion}

The new proposed diagnostic criteria are an international collaboration of pediatric, adult gastroenterologist, allergist-immunologists, pathologists, and researchers. The new criteria allow some flexibility as the clinical presentation and treatments can vary for patients and provider. It allows for adult gastroenterologists to diagnose EoE without a PPI trial and also allows pediatrician to use PPIs to assess abdominal pain related to GERD without diagnosing EoE. It is important to note that these new guidelines are compatible with previous guidelines, in that patients previously diagnosed with EoE will still meet the new diagnostic criteria. For clinical trial participation, PPI trial is no longer mandatory, But, it might be some benefit for some clinical trials to examine and characterize appropriate patient cohorts for investigational medicines. Similar to previous guidelines, there will be a need for education of allergist-immunologist, gastroenterologists, pathologists, patients, and families on these new criteria. There are several obvious potential research and clinical questions that exist, in particular those about the comparative effectiveness of a PPI as a first 
line therapy compared to other modalities such as diet and/or steroid, and if certain patient phenotypes are more responsive to one of these three potential first-line therapies. Finally, research is need to better understand various phenotypes ${ }^{23}$ that can be seen in EoE.

Like all things in medicine and science, new research may lead to changes in these recommendations as additional randomized clinical trials are done to answer these questions.

\section{Acknowledgments}

Financial support:

The International Gastrointestinal Eosinophilic Diseases Researchers (TIGERS), The David and Denise Bunning Family, U54AI1 17804 (CEGIR), which is part of the Rare Disease Clinical Research Network (RDCRN), an initiative of the Office of Rare Disease Research (ORDR), NCATS, and is funded through collaboration between NIAID, NIDDK, NCATS and patient advocacy groups including APFED, CURED, and EFC, and NIH K24DK100303 (GTF)

Disclosures:

Dellon - Consultant - Adare, Allakos, Alivio, Banner, Celgene/Receptos, Enumeral, GSK, Regeneron, Shire; Research funding - Adare, Celgene/Receptos, Miraca, Meritage, Nutricia, Regeneron, Shire; Educational grant Banner, Holoclara Liacouras - Consultant - Shire, Adare, Abbott Nutrition, Receptos, TEVA Molina-Infante. Research funding and Consultant Dr. Falk Pharma Furuta - Founder of EnteroTrack, Consultant for Shire, Royalties from UpToDate Spergel - Consultant-Regeneron, DBV Technology, Kaleo, Grant-DBV Technology, Aimmune Therapeutics, Food Allergy Research Education, Royalities-UpToDate Hirano - Consultant - Adare, Allakos.

\section{References}

1. Furuta GT, Liacouras CA, Collins MH, et al. Eosinophilic esophagitis in children and adults: a systematic review and consensus recommendations for diagnosis and treatment. Gastroenterology. 2007; 133:1342-1363. [PubMed: 17919504]

2. Liacouras CA, Furuta GT, Hirano I, et al. Eosinophilic esophagitis: Updated consensus recommendations for children and adults. J Allergy Clin Immunol. 2011; 128:3-20e26. [PubMed: 21477849]

3. Dellon E, Liacouras C, Molina-Infante J, et al. Updated international consensus diagnostic criteria for eosinophilic esophagitis: Proceedings of the AGREE conference. Gastroenterology. 2018 In Press.

4. Lucendo AJ, Arias A, Molina-Infante J. Efficacy of Proton Pump Inhibitor Drugs for Inducing Clinical and Histologic Remission in Patients With Symptomatic Esophageal Eosinophilia: A Systematic Review and Meta-Analysis. Clin Gastroenterol Hepatol. 2016; 14:13-22.e11. [PubMed: 26247167]

5. Dellon ES, Speck O, Woodward K, et al. Clinical and Endoscopic Characteristics do Not Reliably Differentiate PPI-Responsive Esophageal Eosinophilia and Eosinophilic Esophagitis in Patients Undergoing Upper Endoscopy: A Prospective Cohort Study. Am J Gastroenterol. 2013; 108:18541860. [PubMed: 24145677]

6. Warners MJ, van Rhijn BD, Curvers WL, Smout AJ, Bredenoord AJ. PPI-responsive esophageal eosinophilia cannot be distinguished from eosinophilic esophagitis by endoscopic signs. Eur J Gastroen Hepat. 2015; 27:506-511.

7. Muir AB, Wang ML, Metz D, et al. Proton pump inhibitor-responsive oesophageal eosinophilia: too early to change clinical practice. Gut. 2017; 66:979-980. [PubMed: 27464706]

8. Wen T, Dellon ES, Moawad FJ, Furuta GT, Aceves SS, Rothenberg ME. Transcriptome analysis of proton pump inhibitor-responsive esophageal eosinophilia reveals proton pump inhibitor-reversible allergic inflammation. J Allergy Clin Immunol. 2015; 135:187-197. [PubMed: 25441638] 
9. Shoda T, Matsuda A, Nomura I, et al. Eosinophilic esophagitis versus proton pump inhibitorresponsive esophageal eosinophilia: Transcriptome analysis. J Allergy Clin Immunol. 2017; 139:2010-2013.e2014. [PubMed: 28063872]

10. Sodikoff J, Hirano I. Proton pump inhibitor-responsive esophageal eosinophilia does not preclude food-responsive eosinophilic esophagitis. J Allergy Clin Immunol. 2016; 137:631-633. [PubMed: 26318073]

11. Lucendo AJ, Arias A, Gonzalez-Cervera J, Olalla JM, Molina-Infante J. Dual response to dietary/ topical steroid and proton pump inhibitor therapy in adult patients with eosinophilic esophagitis. J Allergy Clin Immunol. 2016; 137:931-934 e932. [PubMed: 26371836]

12. Cheng E, Zhang X, Huo X, et al. Omeprazole blocks eotaxin-3 expression by oesophageal squamous cells from patients with eosinophilic oesophagitis and GORD. Gut. 2013; 62:824-832. [PubMed: 22580413]

13. Zhang X, Cheng E, Huo X, et al. Omeprazole blocks STAT6 binding to the eotaxin-3 promoter in eosinophilic esophagitis cells. PLoS One. 2012; 7:e50037. [PubMed: 23185525]

14. van Rhijn BD, Weijenborg PW, Verheij J, et al. Proton pump inhibitors partially restore mucosal integrity in patients with proton pump inhibitor-responsive esophageal eosinophilia but not eosinophilic esophagitis. Clin Gastroenterol Hepatol. 2014; 12:1815-1823 e1812. [PubMed: 24657840]

15. Barthel SR, Annis DS, Mosher DF, Johansson MW. Differential engagement of modules 1 and 4 of vascular cell adhesion molecule-1 (CD106) by integrins alpha4beta1 (CD49d/29) and alphaMbeta2 (CD11b/18) of eosinophils. J Biol Chem. 2006; 281:32175-32187. [PubMed: 16943205]

16. Tobey NA, Gambling TM, Vanegas XC, Carson JL, Orlando RC. Physicochemical basis for dilated intercellular spaces in non-erosive acid-damaged rabbit esophageal epithelium. Dis Esophagus. 2008; 21:757-764. [PubMed: 18522636]

17. Dunbar KB, Agoston AT, Odze RD, et al. Association of Acute Gastroesophageal Reflux Disease With Esophageal Histologic Changes. J Amer Med Assoc. 2016; 315:2104-2112.

18. Lucendo AJ, Molina-Infante J, Arias A, et al. Guidelines on eosinophilic esophagitis: Evidencebased statements and recommendations for diagnosis and management in children and adults. United Eur Gastroent. 2017; 5:335-358.

19. Hirano I, Moy N, Heckman MG, Thomas CS, Gonsalves N, Achem SR. Endoscopic assessment of the oesophageal features of eosinophilic oesophagitis: validation of a novel classification and grading system. Gut. 2013; 62:489-495. [PubMed: 22619364]

20. Gonsalves N, Policarpio-Nicolas M, Zhang Q, Rao MS, Hirano I. Histopathologic variability and endoscopic correlates in adults with eosinophilic esophagitis. Gastrointest Endosc. 2006; 64:313319. [PubMed: 16923475]

21. Katz PO, Gerson LB, Vela MF. Guidelines for the diagnosis and management of gastroesophageal reflux disease. Am J Gastroenterol. 2013; 108:308-328. quiz 329. [PubMed: 23419381]

22. Dellon ES, Liacouras CA. Advances in Clinical Management of Eosinophilic Esophagitis. Gastroenterology. 2014; 147:1238-1254. [PubMed: 25109885]

23. Atkins D, Furuta GT, Liacouras CA, Spergel JM. Eosinophilic Oesophagitis phenotypes: Ready for primetime? Pediatr Allergy Immunol. 2017 


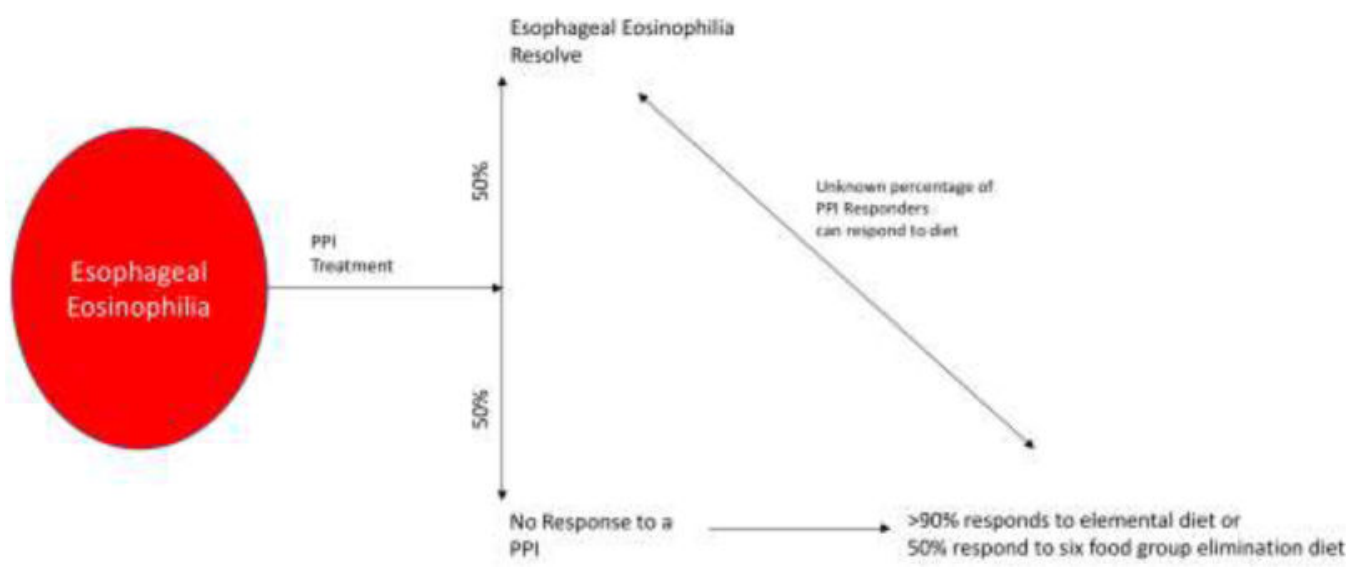

Figure 1. Esophageal Eosinophilia Response

Potential response of subjects with esophageal eosinophilia. In average, $50 \%$ of patients will have improvement with high dose PPI. In the $50 \%$ of patients that do not respond to PPI, almost all (>90\%) will have resolution of esophageal eosinophilia with an elemental diet. In the $50 \%$ of the patients that respond to PPI, the exact percentage that will respond to diet or swallowed steroids is unknown. This is not a proposed treatment pathway, but represents response to therapy for examining the role of antigen and diagnostic criteria. 
Clinical Presentation suggestion of EoE

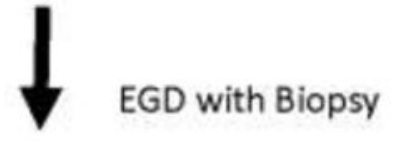

Esophageal eosinophilia $\geq 15$ eos $/ \mathrm{hpf}\left(\approx 60 \mathrm{eos} / \mathrm{mm}^{2}\right)$

Evaluate for non-EoE disorders that cause or

$\checkmark$ potentially contribute to esophageal

eosinophilia

Eosinophilic Esophagitis

Figure 2. Updated EoE diagnostic algorithm

New diagnostic algorithm. Figure reprinted with permission from Gastroenterology. ${ }^{3}$ 


\title{
Table 1
}

\section{EoE diagnostic criteria ${ }^{3}$}

\author{
○ Symptoms of esophageal dysfunction; \\ ○ Concomitant atopic conditions should increase suspicion for EoE \\ ○ Endoscopic findings of rings, furrows, exudates, edema, stricture, narrowing, and crepe-paper mucosa should increase suspicion for EoE \\ ○ $>15$ eos/hpf $(\sim 60$ eos/mm²) on esophageal biopsy \\ $\circ$ Eosinophilic should be isolated to the esophagus
}

Other causes of EoE has been ruled out including but not limited to: Eosinophilic gastritis, gastroenteritis, or colitis with esophageal involvement; Gastroesophageal reflux disease; Achalasia and other disorders of esophageal dysmotility; Hypereosinophilic syndrome; Crohn's disease with esophageal involvement; Infections (fungal, viral); Connective tissue disorders; Dermatologic conditions with esophageal involvement (ie pemphigus); Drug hypersensitivity reactions; Pill esophagitis; Graft vs host disease; Mendelian disorders (Marfan Syndrome Type II, Hyper-IgE Syndrome, PTEN Hamartoma Tumor Syndrome, Netherton’s Syndrome, Severe Atopy Metabolic Wasting Syndrome) 